\title{
A RARE QUADRUPLICATE ARRANGEMENT OF ABDUCTOR POLLICIS LONGUS TENDONS - ANATOMICAL AND CLINICAL RELEVANCE
}

\author{
doi: 10.1590/S1807-59322009000200014
}

Vandana Mehta, Jyoti Arora, Rajesh Kumar Suri, Gayatri Rath

\section{INTRODUCTION}

Movements of the thumb are carried out by extrinsic and intrinsic muscles and have immense phylogenetic and evolutionary significance. The number, length, and thickness of APL accessory tendons may have a functional role in the etiology of DeQuervain's stenosing tenovaginitis. ${ }^{1}$ Thus, precise knowledge of the variations in this region is important for hand surgeons. Additionally, these tendons may prove to be of importance in plastic reconstructive surgeries where they could be utilized as effective transplant tissues. ${ }^{2,3}$ Technological advancements in MRI imaging have enabled hand surgeons to identify the musculo-tendinous and retinacular structures of the dorsum of the hand in detail ${ }^{4}$. Familiarity with the variations in extensor tendons of the hand and fingers is vital for appropriate diagnosis of neurological disorders. Anatomical comprehension is particularly essential for clinical situations related to extensor tendon injuries and inflammatory diseases. The main objective of this report is to illustrate in detail the variant insertion pattern of APL tendon slips and to discuss their functional and clinical relevance.

\section{CASE DISCUSSION}

The APL muscle variant found in the right upper limb belonged to an adult male cadaver. The muscle was well developed and originated from the radius as usual. It ramified into four tendon slips $6.4 \mathrm{~cm}$ proximal to the base of the first metacarpal bone (Figure 1). Strikingly, the intermediate

Vardhman Mahavir Medical College - Anatomy Safdarjung Hospital New Delhi - Delhi-India

Email: drvandanamehta@gmail.com

Tel.: 55 112592-7322 two tendons were of equal length $(4.4 \mathrm{~cm})$, whereas the medial-most and lateral-most tendons were of equal lengths $(3.5 \mathrm{~cm})$. Distally, these tendons were clearly discernible approximately $2 \mathrm{~cm}$ before the insertion.

The medial-most tendon was observed to ramify into two lateral $(1.9 \mathrm{~cm}$ in length) and medial $(1.4 \mathrm{~cm}$ in length) slips, which were attached to the trapezium bone close to the origin of the abductor pollicis brevis. The tendons in the center approached the base of the first metacarpal in the classical manner, justifying their designation as the main APL tendon. The extensor pollicis brevis displayed typical morphology. Innervation of the APL was derived as usual from the posterior interosseous nerve. The left upper limb did not exhibit any obvious muscular variations.

\section{DISCUSSION}

The present report describes an unusual pattern of division of the APL tendons. Although duplication and triplication of APL tendons has been frequently reported, ${ }^{2,5}$ division of the APL into seven tendons was reported for the first time by Melling et al. ${ }^{6}$ In a recent study, six APL tendons were reported in an adult cadaveric specimen; the medial two tendons were inserted into the trapezium and the remaining tendons into the base of first metacarpal bone. The extensor pollicis brevis was absent in this specimen, thus reducing the number of compartments to five. ${ }^{7}$

The uniqueness of the present study stems from the ramification of the APL into four tendons, as well as from the bifurcation pattern of the medial-most tendon. These aberrant tendons are clinically important because their presence can cause persistent pain after surgical division of the first tunnel to treat thumb inflammation. Awareness of APL muscle variants could therefore offer an effective solution for appropriate recognition of these accessory tendons and 
for dividing them together with the main tendon. Clinical experience dictates that these tendons are at an increased risk for injury. DeQuervain's stenosing tenovaginitis is a pathological condition that could be caused by the presence of these supernumerary tendon slips. ${ }^{1}$ Phylogenetically, these APL tendon slips are found in other primates such as gorillas and gibbons, demonstrating an instance of atavism. ${ }^{1,6}$

Presumably, the quadruplicate pattern of the tendon slips found in the present case could represent a recapitulation of this primitive pattern of tendon attachment. The embryological basis of the varied pattern of tendinuous divisions of the APL can be traced to an embryo with a crown-rump length of $20 \mathrm{~mm}$. The differentiating APL tendon is divided into three strips; the middle inserts into the trapezium, the dorsal inserts into the first metacarpal, and the palmar lies in a distal direction, blending with the opponens pollicis. ${ }^{78} \mathrm{New}$ connections may be subsequently established between the palmar strip and adjacent abductor pollicis brevis while the connection with the opponens pollicis is lost. Thus, the multiplicity of tendons may be attributed to the persistence of a tendinuous pattern observed during early fetal life.

These accessory tendons have been found to be a feasible option for tendon transplants in reconstructive surgeries of the hand. ${ }^{2,9}$

Partial subluxation of the trapeziometacarpal joint is an uncommon condition to which patients with Bennett's fracture are predisposed. Absence of insertion of the APL tendon into the first metacarpal bone may result in unbalanced muscle forces, leading to bilateral sub-luxation of the trapezio-metacarpal joint. ${ }^{10}$ These supernumerary APL tendons could be used effectively for supplementing the lax trapeziometacarpal joint, owing to their insertion into the fascia of the APB and trapezium bone. Thus, the presence of these tendons reduces the vulnerability of the patient to such chronic dislocations. The latest technological advancements in radiological procedures, including MRI, detect the presence of multiple extensor tendons ${ }^{4}$. Hence, it becomes mandatory to identify these multiple tendons during exploratory procedures prior to tendon transfer.

Division of the tendons into several parts was observed to result in effective distribution of stress during thumb abduction. ${ }^{11}$ Furthermore, palmar flexion at the wrist is also supported. ${ }^{12}$ Kinematic analysis of thumb reconstruction provided vital information demonstrating that the standard tendon interposition technique produces a larger radius of motion quite similar to that of an intact thumb. ${ }^{13}$ Additionally, modified trapeziectomy application to the APL combined with soft tissue arthroplasty for treatment of carpo-metacarpal arthritis has been shown to improve thumb alignment. ${ }^{14}$ Thus, it is likely that the presence of accessory tendons could confer a biomechanical advantage. A novel surgical trans-osseous approach in the region of the thumb that was developed for ligamentoplasty identified neurovascular structures that are vulnerable to compression. These structures were superficial branches of the radial nerve, radial artery, and endings of the lateral cutaneous nerve of the forearm..$^{15}$ Admittedly, the clinical history of this patient was not available for validation of the findings. Nevertheless, it is well-established that multi-tendoned muscles increase the strength of the thumb. Anatomists believe that these supernumerary tendons augment the functional capacity of APL tendons; thus trauma to a single tendon would not incapacitate the thumb.

\section{CONCLUSIONS}

Multiplicity of APL tendons can be viewed as a functional advantage, since injuries in one tendon can be compensated for by the remaining tendons. Precise knowledge of the various APL tendon morphologies is vital for reconstructive procedures to rehabilitate traumatized hands.

\section{REFERENCES}

1. Bunnel S. Surgery of the hand, $2^{\text {nd }}$ ed,J.B.Lippincott, Philadelphia, 1948, p.455-57.

2. Lacey T II, Goldstein LA, Tobin CE. Anatomical and clinical study of the variations in the insertions of abductor pollicis longus tendon associated with stenosing tenovaginitis. J Bone Jt Surg Am. 1951;33:347-50.

3. Bergman RA, Thompson SA, Affifi AK, Saadeh FA. Compendium of human anatomic variation. Baltimore, Urban \& Schwarzenberg, (1988),p.16.
4. Clavero JA, Golana P, Farinas O, Alomar X, Monill JM, Esplugas M. Extensor mechanism of fingers-MR imaging-Anatomical correlation. Radiographics. 2003;23:593-611.

5. Baba MA. The accessory tendon of the abductor pollicis longus muscle. Anat Rec. 1954;119:541-7.

6. Melling M, Wilde J, Schnallinger M, Schweighart W, Panholzer M. Supernumerary tendons of the abductor pollicis. Acta Anat. 1996;155:291-4. 
7. Nayak SR, Krishnamurthy A, Pai MM, Prabhu LV, Ramanathan LA, Ganesh $\mathrm{Kr} \mathrm{C}$, et al. Multiple variations of extensor tendons of forearm. Rom J Morphol Embryol. 2008;49:97-100.

8. Cihák R. Connection of abductor pollicis longus and abductor pollicis brevis in ontogenesis of human hand. Folia Morphol (praha). 1972;20:102-5.

9. Neviaser RJ, Wilson JN, Gardner MM. Abductor pollicis longus transfer for replacement of first dorsal interosseous. J Hand Surg. 1980;5:5357.

10. Martinez R, Omer GE Jr. Bilateral subluxation of bas eof thumb secondary to an unusual abductor pollicis longus insertion: A case report. J Hand Surg. 1985;10:396-9.

11. Melling M, Reihsner R, Steindl M, Karimian-Teherani D, Schnallinger M, Behnam M. Biomechanical stability of Abductor pollicis longus muscles with variable numbers of tendinuous insertions. Anat Rec. 1998;250:475-9.
12. Kauer JMG. The interdependent carpal articulation chains. Acta Anat 1974;88:481-501

13. Koff MF, Zhao KD, Mierisch CM, Chen MY, An KN, Cooney WP 3rd. The kinematics after thumb carpo-metacarpal joint reconsruction-an in vitro comparison of various constructs. J Hand Surg (Am). 2007;32:68896.

14. Viegas SF. A new modification of trapeziectomy and soft tissue interposition arthroplasty with APL advancement. Tech Hand Up Extrem Surg. 2006;10:130-8.

15. Durand S, Gagey O, Masquelet AC, Thoreux P. Neuro-vascular erlationships of the approaches for arthroscopic total trapeziectomy with ligamentous stabilization. Surg Radiol Anat. 2005;27:165-70. 